\title{
Clinical predictors of functional recovery at six month post-stroke
}

\author{
Caleb Ademola Gbiri1,2, Aderonke O. Akinpelu ${ }^{3}$, Adesola Ogunniyi ${ }^{4}$, Abiodun E. Akinwuntan ${ }^{5}$, \\ C. Werdie Van Staden ${ }^{6}$
}

${ }^{1}$ Lecturer and Neurophysiotherapist, Department of Physiotherapy, College of Medicine, University of Lagos, Idi-Araba, Lagos, Nigeria, ${ }^{2}$ Post-Doctoral Research Fellow, Department of Psychiatry, Faculty of Health Sciences, University of Pretoria, South Africa, ${ }^{3}$ Department of Physiotherapy, College of Medicine, University of Ibadan, Nigeria, ${ }^{4}$ Department of Medicine, College of Medicine, University of Ibadan, Nigeria, ${ }^{5}$ Department of Physical Therapy, Georgia Regents University, Augusta GA, USA, ${ }^{6}$ Department of Psychiatry, University of Pretoria, South Africa, Department of Psychiatry, Faculty of Health Sciences, University of Pretoria, South Africa

\section{A B S TR A C T}

Aim: To investigate predictors of functional recovery at six-month among Nigerians with first-ever stroke. Methods: Participants with first ever stroke were recruited at stroke-onset from the University College Hospital, Ibadan, Nigeria. Stroke severity was measured using the National Institute of Health Stroke-Scale. Stroke was classified using the results of the CT scan of the brain. The weighted-standard values of Barthel Index and Frenchay Activities Index were combined to indicate Comprehensive Activities of Daily Living (CADL). The presence of depressive features and Trunk-Control (TC) were measured using the Centre for Epidemiological Scale-Depression and the Postural Assessment-Scale for Stroke-Patients respectively. Measurements were taken every month for six months. Data were analyzed using multivariate regression and survival analyses at $p=0.05$. Results: Sixty-five participants were recruited. Ten died within a week of stroke onset. Fifty-five (mean age $=57.4 \pm 14.8$ years, 28 males) participants completed the study; Twenty-six (47.3\%) had ischaemic stroke and $29(52.7 \%)$ had haemorrhagic stroke. Forty of the 55 participants were married and of the 40,31 reported spousal support. Type of stroke $(\beta=7.5)$ and age $(\beta=-0.4)$ significantly predicted functional recovery after controlling for co-morbidity $(\beta=-2.1)$, brainstem lesion $(\beta=-0.2)$, stroke severity $(\beta=-0.6)$ and $\mathrm{TC}(\beta=0.7)$ and the scores on depressive symptoms ratings $(\beta=-0.1)$. Conclusion: Functional recovery at six-month is better in individuals who had haemorrhagic stroke. However, functional recovery decreases as age increases. The combination of haemorrhagic stroke with the presence of co-morbidity predicted death after stroke.

Key words: Stroke, Ischaemia, Haemorrhage, Rehabilitation, Functional recovery

\section{INTRODUCTION}

One of the major consequences of stroke is death. Residual disabilities that negatively impact the stroke survivor's functional independence and quality of life are another major consequence. ${ }^{1-10}$ Stroke is one of the leading cause of death and leading cause of disability worldwide. ${ }^{10}$ It is also the most common cause of loss of functional ability among the elderly. ${ }^{10-19}$ Even in developed countries with advanced healthcare systems, $60 \%$ of persons who suffer stroke either die, become permanently disabled, or dependent functionally. ${ }^{9-10}$
Access this article online Website:

http://nepjol.info/index.php/AJMS 
for home adjustments and community support. A better understanding of the factors that predict prognosis and recovery from stroke will potentially assist in designing preventive measures and treatment strategies to improve motor function post-stroke.

Recovery, both neurologically and functionally, after stroke has been shown to be rapid within the first six-month and continues slowly thereafter. ${ }^{21,22}$ Although many factors have been reported to influence functional recovery after stroke, ${ }^{17-19,23,24}$ factors determining functional recovery at the 6-month have not been well established. This is probably due to the fact that most of the previous studies covered at least 14 days after the incidence of stroke $e^{12,13,25-30}$ and the clinical and personal variables of the patients at stroke onset were not considered as determinants in their analysis. In this study, we collected multiple data within 24 hours of onset for 6 months from first ever stroke survivors. The data were analyzed to determine the predictors of functional recovery.

\section{METHODS}

The protocol for this study was ethically cleared and approved by the joint University of Ibadan and University College Hospital Research Ethics Committee in Nigeria. All participants consented before inclusion in the study. For participants who were initially unconscious, consent was initially obtained by proxy from participants' relatives. The processes were repeated after such participant regained consciousness and were capable of giving consent. Participants were recruited consecutively from the accident and emergency unit of UCH. Physiotherapy was provided throughout the study period by one of the authors (CAG) and outcome measurements were taken by a research assistant. Each participant received physiotherapy in a standardized format for six months. Each of the participants was treated daily for the first 14 days of stroke and subsequently at every other day for the 12-month period. Achievement of trunk control both from lying to sitting and in sitting position were pre-requisites for the commencement of standing. The ability to shift weight from one lower limb to the other and the achievement of at least $45 \%$ of body weight distribution on the affected limb were taken as pre-requisites for ambulatory re-education. None of the participants received any other physiotherapy treatment other than the one that was provided by the researcher (CAG). The achievement of active wrist and at least fingers' flexion with or without mild spasticity were pre-requisites to graduate the patient to restraining the unaffected upper limb for between eight to ten waking hours per day to improve functional performance of the affected upper limb. A patient who had commenced ambulation but has not been able to achieve heel-strike and heel-off had 30 minutes of bicycle ergometer training for every other day. When the achievement of heel-strike and heel-off had been achieved with or without the achievement of swing-through, a patient was graduated to 20-30 minutes of treadmill training for three times per week with a least a day interval. The social supports were monitored to prevent over-dependency on the informal caregivers especially after hospital discharge in order to promote functional independence. None of them received physiotherapy from any other source throughout the duration of involvement in the study. Participants were treated daily for the first 14 days of stroke and subsequently at every other day for a six-month period. Treatments were individualized and progression was based on the achievement of the pre-determined goals or outcomes. Patient was progressed to the next stage of functional training if they matched with or surpassed the expected outcomes. Social support was monitored to avoid additional physiotherapy-related interventions to promote functional independence from informal caregivers, especially after hospital discharge. The presence and quality of social support received by each patient was measured on a visual analogue scale (scale ranged between 0 and 10).

Stroke severity was measured using US-National Institute of Health Stroke-Scale. ${ }^{31}$ Participants were interviewed for socio-demographic data at when they became stable and alert. The presence and type of co-morbidities were also documented. The stroke types were classified using the clinical indices ${ }^{3,24}$ and the results of brains' computed topography (CT) scan. Urinary incontinence was defined as the report of urinary accidents (wetting of bed or cloth) or the presence of an indwelling catheter. Bowel incontinence was defined as report of faecal accidents. Barthel Index ${ }^{32}$ and Frenchay Activities Index ${ }^{33}$ were administered monthly for 6 -month post-stroke and their weighted-standardized scores were combined to indicate functional recovery. Depression features and Trunk-Control were measured fortnightly for 6-month using the Centre for Epidemiological Scale-Depression ${ }^{34}$ and the Postural Assessment-Scale for Stroke-Patients ${ }^{35}$ respectively. Data were analyzed using descriptive statistics, linear regression and survival analyses $(p=0.05)$.

\section{RESULTS}

Sixty-five participants were recruited into this study (33 males and 32 females), 10 participants, all with history of diabetes mellitus and had haemorrhagic stroke, died within two-week of stroke onset. Fifty-five (28 males and 27 females) participants with a mean age of $57.4 \pm 14.8$ years completed all aspects of this study. 
Thirty (54.6\%) participants had at least one co-morbid condition with diabetes mellitus been the most common (49.1\%), followed by coronary heart disease (20\%), obesity $(1.5 \%)$ and HIV / AIDS (1.5\%) respectively. Details of the other clinical history of the participants are presented in Table 1. Fifty-one (92.7\%) participants had high blood pressure at stroke onset irrespective of their type of stroke. Those who suffered hemorrhagic stroke had systolic and diastolic blood pressure readings of $198 \pm 15.6 \mathrm{mmHg}$ and $120 \pm 18.1 \mathrm{mmHg}$ respectively at stroke onset. They also had blood glucose level of $(172 \pm 23.4)$ and $(85 \pm 13.1)$ at stroke onset and 24 hours post-stroke even in those with no previous history of diabetes mellitus. Fifty-two (94.6\%) had bladder incontinence while $58.2 \%$ had both bowel and bladder incontinence.

The marital statuses of the 55 participants who completed the study are presented in Table 2. Three, 40, 1 and 11 participants were single, married, separated and widowed respectively. Of the 40 married participants, 23 reported having spousal support. Detailed information on other demographic characteristics including type of marriage, highest educational qualification, and occupational status are also reported in Table 2.

At 6-month post-stroke, the functional performance was significantly higher $(p<0.001)$ among (i) the participants without co-morbidity $(76.2 \pm 14.1)$ than those with co-morbidity (59.3 \pm 11.1 ), (ii) those who suffered haemorrhagic stroke $(77.2 \pm 16.2)$ than those who suffered ischaemic stroke (60.3 \pm 15.2$)$, and (iii) those with educational qualifications above secondary school (73.3 \pm 10.2$)$ level than those with secondary education and below (60.3 \pm 15.2$)$. Each of Trunk Control $(\mathrm{r}=0.56)$, stroke severity $(\mathrm{r}=-0.48)$, depression scores $(r=0.50)$ and age $(r=-0.69)$ significantly correlated with functional performance at 6-month. Details of the correlation coefficients of all the variables with functional performance at 6-month are presented in Table 3.

\begin{tabular}{lcc}
\multicolumn{3}{l}{ Table 1: Clinical history of the participants } \\
\hline Variable & $\mathbf{N}$ & Percentage \\
\hline Type of stroke & & \\
$\quad$ Ischaemic stroke & 26 & 47.2 \\
$\quad$ Hemorrhagic stroke & 29 & 52.7 \\
$\quad$ Side of the brain affected & & \\
$\quad$ Left & 44 & 80 \\
$\quad$ Right & 11 & 20 \\
Speech problem & & \\
$\quad$ Aphasia & 11 & 20 \\
$\quad$ Dysartheria & 11 & 20 \\
Severity of stroke & & \\
$\quad$ Moderate & 15 & 27.3 \\
$\quad$ Severe & 40 & 72.7 \\
Location of brain lesion & & \\
$\quad$ Cortex & 53 & 96.4 \\
$\quad$ Sub-cortex & 2 & 3.6 \\
\hline
\end{tabular}

None of sex, side of lesion, occupational status, limb dominance, blood pressure and glucose level at the onset of stroke or stroke severity predicts functional recovery at 6-month post-stroke ( $\mathrm{p}>0.05)$.

Age $(\beta=-0.4)$ and type of stroke (with those who had haemorrhagic stroke having better outcome $)(\beta=7.5)$ were the most important predictors of functional performance at 6-month post-stroke after controlling for marital status

\begin{tabular}{|c|c|c|}
\hline Variables & $\mathbf{N}$ & Percentage \\
\hline \multicolumn{3}{|l|}{ Age group } \\
\hline 20-39 & 5 & 9.09 \\
\hline $40-59$ & 23 & 41.82 \\
\hline $60-79$ & 25 & 45.46 \\
\hline 80-89 & 2 & 3.63 \\
\hline \multicolumn{3}{|l|}{ Marital status } \\
\hline Singled & 3 & 5.5 \\
\hline Married & 40 & 72.7 \\
\hline Widowed & 11 & 20.0 \\
\hline Separated & 1 & 1.8 \\
\hline \multicolumn{3}{|l|}{ Type of marriage } \\
\hline Monogamy & 23 & 57.5 \\
\hline Polygamy & 17 & 42.5 \\
\hline \multicolumn{3}{|l|}{ Spouse support } \\
\hline Not applicable & 15 & 5.5 \\
\hline Spouse support & 22 & 76.4 \\
\hline No spouse support & 18 & 18.2 \\
\hline \multicolumn{3}{|c|}{ Highest educational qualification } \\
\hline No-formal & 10 & 18.2 \\
\hline Primary & 6 & 10.9 \\
\hline Secondary & 25 & 45.5 \\
\hline Post-secondary & 4 & 7.2 \\
\hline University & 10 & 18.2 \\
\hline \multicolumn{3}{|l|}{ Occupational status } \\
\hline Students & 3 & 5.5 \\
\hline Non-skilled & 16 & 29.1 \\
\hline Semi-Skilled & 17 & 30.9 \\
\hline Skilled & 8 & 14.6 \\
\hline Professional & 4 & 7.3 \\
\hline Retiree & 3 & 5.5 \\
\hline Unemployed & 3 & 5.5 \\
\hline
\end{tabular}

\begin{tabular}{lcc} 
Table 3: Relationship between \\
socio-demographics and clinical predictors of \\
post-stroke functional recovery at 6-month \\
\hline Variables & $\mathbf{r}$ & $\mathbf{p}$-Value \\
\hline Marital status & -0.45 & 0.01 \\
Educational qualification & 0.45 & 0.01 \\
Trunk control & 0.56 & 0.04 \\
Incontinence & 0.57 & 0 \\
Depression & 0.5 & 0.02 \\
Type of stroke & 0.65 & 0 \\
Location of brain lesion & 0.52 & 0.04 \\
Co-morbidity & 0.56 & 0.02 \\
Age & -0.69 & 0 \\
Spousal support & 0.46 & 0 \\
Stroke severity & -0.48 & 0 \\
\hline p<0.05 & &
\end{tabular}


$(\beta=1.2)$, educational level $(\beta=0.2)$, presence or absence co-morbidity health condition $(\beta=-2.1)$, presence of brainstem lesion $(\beta=-0.2)$, stroke severity $(\beta=-0.6)$, and trunk control performance $(\beta=0.7)$ and depression scores $(\beta=-0.1)$ at 2 -week post-stroke. Age contributed $48 \%$ while type of stroke contributed $42 \%$ to post-stroke functional recovery (Table 4). The combination of haemorrhagic stroke with the presence of co-morbid health conditions especially diabetes mellitus predicted death within 2 -week of stroke.

\section{DISCUSSION}

This study investigated the clinical predictors of clinical predictors of functional recovery at 6 months post-stroke in first-ever stroke survivors. The study involved stroke patients who had suffered the first-ever stroke. This was done to eliminate the effect of residual disability that might be present in stroke patients with recurrent stroke episode.

Diabetes mellitus was the most common co-morbid health problem found among the participants. Diabetes has been shown to be as a major risk factor to stroke. ${ }^{22}$ Fortunately, diabetes especially the adult-onset type can be modified by lifestyle adjustment and exercise. ${ }^{42}$ There is need to improve on public awareness on lifestyle adjustment and modification among Nigerians in order to stem-down stroke incidence. The blood sugar levels of all the participants who had hemorrhagic stroke were higher than normal level at onset of stroke, even though many of them were not known to have diabetes mellitus before the stroke. This shows that high blood sugar is consistent with haemorrhagic stroke at the onset of stroke. Therefore, care should be taken in concluding the diabetic status of stroke patients at the onset of stroke especially those who had suffered haemorrhagic stroke and calls for extra caution on early control blood sugar of stroke patients in order to prevent the patient from retrogressing into hypoglycaemia which could be detrimental to their survival and subsequent functional recovery.

The results of this study indicate that functional recovery post-stroke is not influenced by either the blood pressure or blood glucose level at the onset of stroke. However, those who were documented to have been diagnosed of diabetes mellitus had higher chance of death when they suffered haemorrhagic stroke. This is evident as all the ten participants who died had history of diabetes mellitus and then suffered haemorrhagic type of stroke. This finding suggests that the risk of death during immediate post-stroke period is high among individuals who suffer haemorrhagic stroke and have had previous history of diabetes mellitus.

The result that age and type of stroke (with those who had haemorrhagic stroke having better outcome) were the major predictors of post-stroke functional recovery contributing high percentages shows that age and type of stroke are the major predictors of prognosis in rehabilitation after stroke. However, this result shows that haemorrhagic stroke predicts death in stroke patients at immediate post-stroke period. Although, patients who suffered haemorrhagic stroke are likely to die within few weeks of stroke, they have better outcome in functional recovery if they survive. It was observed that as age increases, the tendency to recover into pre-stroke functional status decreases. This may be attributed to the age related changes in the nervous system of the elderly which are not common in the younger age. ${ }^{25}$

Depression is associated with many chronic and disabling illnesses including stroke. ${ }^{35-37}$ This may be due to the fact that the onset of stroke is sudden and it is usually associated with emotional problems (depression, anxiety and sometimes some personality disorders. ${ }^{12,35-38}$ These factors frequently have negative effects on the emotional and psychological

\begin{tabular}{|c|c|c|c|c|c|c|c|}
\hline \multirow[t]{2}{*}{ Model } & \multicolumn{5}{|c|}{ Standardized coefficients } & \multicolumn{2}{|c|}{$95 \%$ confidence interval for B } \\
\hline & B & Standard error & Beta & t-value & p-value & Lower bound & Upper bound \\
\hline Constant & 100.08 & 20.25 & & 4.94 & 0.00 & 59.21 & 140.95 \\
\hline Marital status & 1.59 & 3.42 & 0.07 & 0.47 & 0.64 & -5.30 & 8.49 \\
\hline Educational level & -0.16 & 1.12 & -0.12 & -0.14 & 0.89 & -2.41 & 2.01 \\
\hline Trunk control score & 0.72 & 0.53 & 0.14 & 1.39 & 0.17 & -0.33 & 1.79 \\
\hline Presence of incontinence & 1.39 & 3.45 & 0.05 & 0.40 & 0.69 & -5.57 & 8.35 \\
\hline Depression score & 0.05 & 0.13 & 0.04 & 0.36 & 0.72 & -0.21 & 0.30 \\
\hline Type of stroke & 7.48 & 3.26 & 0.30 & 2.29 & 0.03 & 0.89 & 14.06 \\
\hline Location of brain lesion & -0.20 & 0.45 & -0.05 & -0.45 & 0.66 & -1.11 & 0.71 \\
\hline Presence of co-morbidity & -2.14 & 3.02 & -0.07 & -0.71 & 0.48 & -8.23 & 3.95 \\
\hline Age & -0.44 & 0.12 & -0.49 & -3.76 & 0.00 & -0.68 & -0.20 \\
\hline Spousal support & 0.50 & 0.61 & 0.12 & 0.82 & 0.42 & -0.73 & 1.72 \\
\hline Stroke severity & -0.59 & 0.51 & -0.15 & -1.16 & 0.25 & -1.60 & 0.44 \\
\hline
\end{tabular}


well-being of stroke survivors. However, the positive association of availability of spousal support with increase in functional recovery may have been related to ameliorating of depression through the provision of spousal support for those who were married. This finding corroborates that of Gbiri et $\mathrm{al}^{3}$ who concluded that spouse support helps alleviate emotional problem, reduces sense of stigmatization and makes a stroke survivor happy. Also, the higher chance of people with higher educational attainment to recover functional status could be attributed to knowledge. Education is expected to inform better knowledge which would translate to increased goal and aspiration.

That severity of stroke significantly influences recovery of functional activities of stroke survivors and that the combination of stroke severity and presence of co-morbidity did not only influence recovery but predicts death in stroke survivors may be attributed to the presence of aphasia as the present of co-morbidity alone was a weak determinant of functional recovery. Sensory deficit, aphasia and dysarthria contributed to the stroke severity in the participants in this study. This may be due to the fact that aphasia and dysarthria may be associated with emotional expression and possible depression, anxiety and frustration. An individual who sustains a left-brain stroke may have inability to solve problems, is often more easily angered and frustrated, has impaired retention of information, and may have language difficulties or apraxia. Language difficulties may translate to difficulty in understanding directions for the therapy, or these individuals may be limited in keeping track of their home activity. With communication difficulty, there may be increased levels of frustration. Those with apraxia may have spatial or timing errors, such as a delay in initiation or inappropriate pauses. ${ }^{37}$

The significant influence of trunk-controls on functional recovery post-stroke shows that if effort is paid to trunk-control in stroke rehabilitation programme, much would be achieved in terms of functional recovery. Therefore, clinicians and healthcare providers in the field of rehabilitation should not trivialize trunk-control's contribution to total recovery of comprehensive function in stroke patients. It should attract much attention during the rehabilitation process. The finding that location of brain lesion influenced functional recovery post-stroke can be attributed to functionality of the brain. This corroborates previous opinion that autonomic function is usually impaired in stroke survivors. ${ }^{45}$ Various clinical and socio-demographic variables that influence the stroke survivors' functional recovery made prediction of prognosis in this set of population more complex as no single factor can be isolated for projecting the outcome of prognosis. Therefore, clinicians should consider every determinant during assessment of a stroke victim.

\section{CONCLUSION}

Functional recovery at six-month is better in individuals who had haemorrhagic stroke. However, functional recovery decreases as age increases. The combination of haemorrhagic stroke with the presence of co-morbidity predicted death after stroke. There is positive association between functional performance at 6-month post-stroke and each of degree of trunk control, availability of spousal support for those were married, absence of incontinence and levels of educational attainment while the degrees of stroke severity and depression had negative and presence of co-morbid health problem had negative correlation with functional performance. Neither gender, side of affectation, occupational status, limb dominance, blood pressure and glucose level at the onset of stroke nor stroke type predicts functional recovery at 6-month post-stroke.

\section{REFERENCES}

1. World Health Organization. World Health Organization task force on stroke and other cerebrosvascular disorders. Recommendation on stroke prevention, diagnosis and therapy. Stroke 1999;20:1407-1431.

2. Teasell R, Bayona N, Salter K, Hellings C and Bitensky J. Progress in Clinical Neuroscience: Stroke Recovery and Rehabilitation. The Canadian Journal of Neurological Science 2006;33:357-364.

3. Gbiri CA, Akinpelu AO and Odole AC. Prevalence, Pattern and Impact of Depression on Quality of Life of Stroke Survivors. International Journal of Psychiatry in Clinical Practice 2010;14:198-203.

4. Akinpelu AO and Gbiri CA. Quality of life of Stroke Survivors and Apparently Healthy Individuals in South-western Nigeria. Physiotherapy Theory and Practice 2009; 25:14-20.

5. Gbiri CA and Akinpelu AO. Relationship between post-stroke functional recovery and quality of life among Nigerian stroke survivors. Nigerian Postgraduate Medical Journal 2013; 20:29-33.

6. Gbiri CA and Akinpelu AO. Influence of motor performance and post-stroke duration on quality of life of stroke survivors. Journal of Clinical Sciences 2012; 9:13-17.

7. Akinpelu OA, Gbiri CA and Maruf FA. Quality of life, Disablement, Co-morbidity and Socio-demographics of Stroke Survivors in South-Western Nigeria. Indian Journal of Physiotherapy and Occupational Therapy 2012; 6:13-18.

8. Gbiri CA and Akinpelu AO. Quality of life of Nigerian stroke survivors during first 12 months post-stroke. Hong Kong Journal of Physiotherapy 2012;30:18-24.

9. Mackay J and Mensah $\mathrm{G}$. The atlas of heart disease and stroke. Geneva, Switzerland: World Health Organisation. 2004.

10. Murray CJL, Vos T, Lozano R, Naghavi M, Flaxman AD, Shibuya K, et al. Disability-adjusted life years (DALYs) for 291 diseases and injuries in 21 regions, 1990-2010: a systematic analysis for the global burden of disease study. The lancet 2012;380(9859):2197-2223.

11. American Heart Association. Heart Disease and Stroke Statistics. 2008.

12. Ostwald SK. Predictors of life satisfaction among stroke survivors and spousals' caregivers: a narrative review. Aging Health 2008;4:241-252. 
13. Stineman MG, Maislin G, Fiedler RC and Granger CV. A prediction Model for functional recovery in stroke. Stroke 1997;28:550-556.

14. Kwakkel G, Kollen B and Wagennar R. Therapy impact on functional recovery in stroke rehabilitation: a critical review of the literature. Physiotherapy 1999;13:457-470.

15. Bagg S, Pombo AP and Hopman W. Effect of age on functional outcomes after stroke rehabilitation. Stroke 2002;33:179-185.

16. Di Carlo A, Lamassa M, Baldereschi M, Pracucci G, Basile AM, Wolfe CDA, et al. Sex differences in the clinical presentation, resource use, and 3-month outcome of acute stroke in Europe: data from a multicenter multinational hospital-based registry. Stroke 2003;34:1114-1119.

17. Hillen T, Davies S, Rudd AG, Kieselbach T and Wolfe CD. Self ratings of health predict functional outcome and recurrence free survival after stroke. J Epid Com Health 2003;57:960-966.

18. Lai SM, Duncan PW, Dew P and Keighley J. Sex differences in stroke recovery. Prevention of Chronic Diseases 2005; [serial online] July.

19. Kollen B, Kwakkel $G$ and Lindeman E. Functional recovery after stroke: A review of current development in rehabilitation research. Review of Recent Clinical Trials 2006;1:75-80.

20. Lin JH, Hsueh IP, Sheu CF and Hsieh CL. Psychometric properties of the sensory scale of the Fugl-Meyer Assessment in stroke patients. Clin Rehab 2004;18:391-397.

21. Teasell R and Foley N. Recovery post-stroke. Evidence-Based Reviewed of Stroke Rehabilitation Module 3, 2004.

22. Hsieh CL, Sheu CF, Hsueh IP, Hsieh and Wang CH. Trunk control as an early determinant of Activities of daily living in stroke patients. Stroke 2002; 33:2626-2630.

23. Gbiri $\mathrm{CA}$ and Akinpelu AO. Pattern of post-stroke functional recovery among Nigerian stroke survivors in the first 12 months. Nigerian Quarterly Journal of Hospital Medicine 2011; 21: 245-248.

24. Teasell R, Bayona N, Salter K, Hellings C and Bitensky J. Progress in Clinical Neuroscience: Stroke Recovery and Rehabilitation. The Canadian Journal of Neurological Sciences 2006;33:357-364.

25. Rameezan BA and Zaliha O. Functional status of acute stroke patients in University Malaya Medical Centre (UMMC), Kuala Lumpur, Malaysia. Medical Journal of Malaysia 2005; 60(5):548-559.
26. Franchignoni FP, Tesio L, Ricupero $\mathrm{C}$ and Martino MT. Trunk control test as an early predictor of stroke rehabilitation outcome. Stroke 1993;28:1382-1385.

27. Feigin L, Sharon B, Czaczkes B and Rosin AJ. Sitting equilibrium 2 weeks after a stroke can predict the walking ability after 6 months. Gerontology 1996;42:348-353.

28. Tilling K, Sterne JAC, Rudd AG, Glass TA, Wityk RJ and Wolfe CDA. A New Method for Predicting Recovery after Stroke. Stroke 2001; 32:2867.

29. Duarte E, Marco E, Muniesa JM, Belmonte R, Diaz P, Tejero M, et al. Trunk control test as a functional predictor in stroke patients. Journal of Rehabilitation Medicine 2002;34:267-272.

30. Fritz SL, Light KE, Clifford SN, Patterson TS, Behrman AL and Davis SB. Descriptive Characteristics as Potential Predictors of Outcomes Following Constraint-Induced Movement Therapy for People After Stroke. Phys Ther 2006; 86:825-832.

31. The National Institute of Health Stroke Scale Know stroke, know the signs, and act in time. National Institute of Neurological Disorders and Stroke. 2007. www.nids.nih.gov. Retrieved 12/07/2007, 12,32hrs.

32. Collin C, Wade DT, Davies S and Horne V. The Barthel ADL Index: a reliability study. International Disability Study 1988;10:61-63.

33. Piercy $\mathrm{M}$, Carter $\mathrm{J}$, Mant $\mathrm{J}$ and Wade DT. Inter-rater reliability of Frenchay Activities Index in patients with stroke and their careers. Clinical Rehabilitation 2000;14:433-440.

34. Geisser ME and Roth RS. Assessing depression among persons with chronic pain using the Center for Epidemiological Studies-Depression Scale and the Beck Depression Inventory: A comparative analysis. Clinical Journal of Pain 1997; 13: 163-70.

35. Franchignoni FP. Psychometric properties and practical attributes of the trunk control test in stroke patients [letter to the editor]. Journal of Rehabilitation Medicine 2003; 35:150.

36. Heilman KM, Watson RT and Valenstein E. Neglect and Related Disorders. Clinical Neuropsychology New York, NY: Oxford University Press, 1993; 279-386.

37. McLaren C, Kerr S, Allan L, Steen IN, Ballard C, Allen J, Murray A and Kenny RA. Autonomic Function Is Impaired in Elderly Stroke Survivors. Stroke 2005:36:1026.

38. Lee CD, Folsom AR and Blair SN. Physical activity and stroke risk. A meta-analysis. Stroke 2003; 34:2475-2482.

\footnotetext{
Authors Contribution:

CAG - Conceptualization, Treatment of Patients, Data Collection, Data analysis and Scientific manuscript writing; AOA - Conceptualization, Data Collection, Data analysis and Scientific manuscript writing; AO - Conceptualization, Data analysis and Scientific manuscript writing; AEA - Data analysis and Scientific manuscript writing; CWVS - Data analysis and Scientific manuscript writing
}

Source of Support: Nil, Conflict of Interest: None declared. 